\title{
Spin and Current Correlation Functions in the d-density Wave State of the Cuprates
}

\author{
Sumanta Tewari, Hae-Young Kee, Chetan Nayak, and Sudip Chakravarty \\ Physics Department, University of California, Los Angeles, CA 90095-1547
}

(October 27, 2018)

\begin{abstract}
We calculate the spin-spin and current-current correlation functions in states exhibiting $d_{x^{2}-y^{2-}}$ density wave (DDW) order, $d_{x^{2}-y^{2}}$ superconducting order (DSC), or both types of order. The spin-spin correlation functions in a state with both DDW and DSC order and in a state with DDW order alone, respectively, illuminate the resonant peak seen in the superconducting state of the underdoped cuprates and the corresponding feature seen in the pseudogap regime. The current-current correlation function in a state with both DDW and DSC order evinces a superfluid density with doping dependence which is consistent with that of the underdoped cuprates. These calculations strengthen the identification of the pseudogap with DDW order and of the underdoped cuprates with a state with both DDW and DSC order.
\end{abstract}

PACS numbers:

\section{INTRODUCTION}

Recently, a new order termed $d$-density wave (DDW) was proposed for the underdoped regime of the high- $T_{c}$ cuprate superconductors [1] 3 . It was argued that the observed anomalies in the pseudogap gap phase are due to the DDW and its competition with the $d$-wave superconductor (DSC). Recent elastic neutron scattering [ 4 , $\mu \mathrm{SR}$ [5], and NMR/NQR [6] experiments appear to have found some direct evidence for the existence of DDW order in YBCO by observing the magnetic fields associated with this order parameter - in other words, by measuring the order parameter itself. In this paper, we wish to elucidate the nature of the competition between the DDW and DSC order paramters by explicitly computing the spin-spin and the current-current correlation functions in two space-dimensions in the Hartree-Fock approximation. Our aim is to provide complementary, indirect evidence in favor of the DDW scenario by showing in some detail that it provides a consistent explanation of the existence and behavior of the resonant peak seen in inelastic neutron scattering and also of the evolution of the superfluid density with doping and temperature, at low temperatures.

Since we deal with broken symmetry states with order parameters, it is possible to argue that their descriptions are adequately described by the Hartree-Fock approximation, at least deep within the ordered phases at zero temperature. The Hartree-Fock approximation by itself cannot be adequate if collective modes are important, but these modes could be described in terms of small oscillations about the ordered state by suitable random phase approximations (RPA). However, critical fluctuations close to a quantum critical point cannot be treated by either Hartree-Fock or RPA. Nonetheless, it is useful to explore the consequences of the simplest possible Hartree-Fock and RPA theories to see if any robust features arise due to the existence of the new order parameter, DDW, and its competition with DSC.
For underdoped bilayer superconductors [7], the dynamic spin-spin correlation function is peaked above $T_{c}$ at the in-plane wavevector $\mathbf{Q}=(\pi, \pi)$ (in this paper we shall take the lattice constant to be unity and will also set $\hbar=c=k_{B}=1$ ) in both even and odd channels with respect to the layers in the bilayer complex. Below $T_{c}$, there is a resonant peak at the same wavevector and approximately the same energy in the odd channel. A resonant peak is also found below $T_{c}$ in optimal and overdoped bilayer high- $T_{c}$ superconductors. There are many approximate theoretical calculations of the dynamic structure factor [8]. Here we concentrate only on the aspects germane to our discussion of DDW. We wish to stress that this analysis is simply a consistency check of the DDW; we do not claim that our explanation of the resonant peak in inelastic neutron scattering is unique or better than the others. The real test of the DDW hypothesis is the direct observation of the order parameter referred to earlier 田 6 . We find that the experimental observations of the neutron dynamic structure factor are consistent with the hypothesis that the neutron scattering peak - and, hence, the pseudogap - is due to DDW order, while the resonance peak in the underdoped superconducting state is due to the coexistence of DDW and DSC orders [3]. The suppression of the resonant peak by a magnetic field perpendicular to the plane suggests that superconducting pairing is important for its formation [9].

The behavior of the superfluid density in the underdoped regime of the high- $T_{c}$ materials is another interesting physical quantity. Many experiments [10] have indicated a rapid collapse of the zero-temperature superfluid density as the doping is decreased below optimal. The zero-temperature penetration depth, for example, grows rapidly in the pseudogap regime, correlating with the suppression of $T_{c}$ with underdoping, yet saturates at overdoping in a way reminiscent of a traditional BCS superconductor [11]. This, as we will show, can be explained by the competition between DDW and DSC orders. At finite temperatures the superfluid density is sup- 
pressed from its zero temperature value at that doping fraction. This suppression is linear in temperature $(T)$ at asymptotically low temperatures, with a slope that is independent of doping. This behavior is captured in our calculations by the thermal excitation of nodal quasiparticles into the upper quasiparticle band. For heavily underdoped samples, there is an intermediate low temperature regime in which we predict this suppression to be proportional to $\sqrt{T}$. There is some experimental evidence for this behavior [12] in $\mathrm{YBa}_{2} \mathrm{Cu}_{4} \mathrm{O}_{8}$, although the explanation of these authors involves a proximity model of alternating stacked superconducting and normal layers. Our analysis is simpler and follows from the nodal exciations in the mixed DDW and DSC state. This prediction can be tested in future experiments in the regime in which pseudogap dominates the DSC gap.

\section{ORDER PARAMETER}

Our Hartree-Fock analysis of the physical quantities merely requires us to specify a mean field Hamiltonian with the proposed order parameters. The actual microscopic Hartree-Fock analyses to obtain these order parameters have been discussed in the past on numerous occasions and need not be repeated here; for a recent set of calculations, see Refs. [2], [13], and references therein. Hamiltonians with short range repulsion and superexchange have the DDW ordered state (both the singlet and the triplet variety) as one of their many possible saddle points. If one includes correlated hopping terms, then within a reasonable range of parameters, the DDW saddle point can be stabilized over the other possibilities. In the present paper, we shall parametrize the order parameters phenomenologically, and our main conclusions are independent of any microscopic self-consistent HartreeFock equations.

The singlet DDW state is defined by the order parameter [2]

$$
\left\langle c_{\mathbf{k} \alpha}^{\dagger} c_{\mathbf{k}+\mathbf{q} \beta}\right\rangle=i \frac{\Phi_{\mathbf{q}}}{2}\left(\cos k_{x}-\cos k_{y}\right) \delta_{\alpha \beta},
$$

where $c_{\mathbf{k} \alpha}$ is the fermion destruction operator for wavevector $\mathbf{k}$ and spin-index $\alpha$, and $\mathbf{q}$ is the ordering wavevector. This order parameter is a generalization of the familiar charge density wave (CDW) order parameter to the case of angular momentum two, in exact analogy to the generalization of the BCS $s$-wave superconducting order parameter to its $d$-wave (DSC) counterpart.

For $\mathbf{q}=\mathbf{Q}$, the case of interest to us, the underlying bipartite square lattice band structure is equivalent under the transformation $\mathbf{Q} \rightarrow-\mathbf{Q}$ and this forces the DDW order parameter to be imaginary [2]. Thus, timereversal symmetry is broken (i.e. the system exhibits magnetism), and the ground state has an array of bond currents, which alternate in direction (clockwise, anticlockwise) in the neighboring plaquettes of the $2 \mathrm{D}$ bipartite square lattice. The corresponding $s$-wave CDW order parameter is, of course, real.

\section{SPIN DYNAMICS}

\section{A. Pure DDW state}

The Hartree-Fock DDW Hamiltonian, using (1), is

$$
H_{\mathrm{DDW}}=\sum_{\mathbf{k} \sigma}\left(\epsilon_{\mathbf{k}}-\mu\right) c_{\mathbf{k} \sigma}^{\dagger} c_{\mathbf{k} \sigma}+\sum_{\mathbf{k} \sigma} i W_{\mathbf{k}} c_{\mathbf{k} \sigma}^{\dagger} c_{\mathbf{k}+\mathbf{Q} \sigma}+\text { h.c. }
$$

where the DDW gap is given by

$$
W_{\mathbf{k}}=\frac{W_{0}}{2}\left(\cos k_{x}-\cos k_{y}\right),
$$

$\epsilon_{\mathbf{k}}=-2 t\left(\cos k_{x}+\cos k_{y}\right)$ gives the band structure, and $\mu$ is the chemical potential. A more realistic band structure would include the effect of next-neighbor hopping $t^{\prime}$. This does not affect our results here, so we drop it for simplicity. At half filling, the chemical potential $\mu=0$, while $\mu$ takes non-zero negative values as we introduce holes into the system.

At $\mu=0$ the zero-temperature spin-spin correlation function $\mathcal{S}$, at momentum transfer $\mathbf{q}=\mathbf{Q}$, is given by

$$
\mathcal{S}(\mathbf{Q}, \omega, \mu=0)=3 \pi \sum_{\mathbf{k} \in \mathrm{rbz}} \delta\left(\omega-2 \sqrt{W_{\mathbf{k}}^{2}+\epsilon_{\mathbf{k}}^{2}}\right),
$$

where the integration is over the magnetic or reduced Brillouin zone (rbz). This function is peaked at an energy equal to twice the maximum value of the gap, $2 W_{0}$. As we dope holes into the system, at nonzero values of $\mu$, Eq. A is changed to

$$
\begin{array}{r}
\mathcal{S}(\mathbf{Q}, \omega, \mu)=3 \pi \sum_{\mathbf{k} \in \mathrm{rbz}} \delta\left(\omega-2 \sqrt{W_{\mathbf{k}}^{2}+\epsilon_{\mathbf{k}}^{2}}\right) \\
\times \theta\left(\mu+\sqrt{W_{\mathbf{k}}^{2}+\epsilon_{\mathbf{k}}^{2}}\right)
\end{array}
$$

The only effect of $\mu$ is the depletion of the integration region. Hence, as long as $|\mu|<W_{0}$, the peak at $\mathbf{q}=\mathbf{Q}$ exists and stays at the same energy $2 W_{0}$.

Note that $\mathcal{S}(\omega)$ also shows a peak for momentum transfer $\mathbf{q}=(\pi, 0)$ 14] at the Hartree-Fock level. From a relation analogous to that of Eq. 5, we can see that the peak-energy for $\mathbf{q}=(\pi, 0)$ scales with $t$. This is much higher in energy than the $(\pi, \pi)$ peak; it is likely to have an enormous width (since there is an enormous amount of phase space into which it can decay) and be unobservable. This is important because no peak has been 
observed at $\mathbf{q}=(\pi, 0)$ in the spin-fluctuation spectrum of the pseudogap regime of the high- $T_{c}$ materials. Early mean-field decouplings of the Heisenberg model 114 led to an effective $t \sim J$, so that the peaks at $\mathbf{q}=(\pi, \pi)$ and $\mathbf{q}=(\pi, 0)$ were comparable in energy, in contradiction to the experiments.

\section{B. Pure DSC state}

Below the superconducting transition temperature $T_{c}$, a DSC state is defined by the gap

$$
\Delta_{\mathbf{k}}=\frac{\Delta_{0}}{2}\left(\cos k_{x}-\cos k_{y}\right)
$$

corresponding to the DSC order parameter. This order parameter, as stressed in reference [1], can compete and coexist with the singlet DDW order parameter. In fact, at half filling, the system can be rotated continuously from a pure DSC order to a pure singlet DDW order and vice versa, without ever having to close the quasi-particle gap [1]. (The symmetry between the $s$-wave counterparts of these two types of orders is exact in the negative $U$ Hubbard model [15.) is

Using (6), the mean-field one-body DSC Hamiltonian

$$
H_{\mathrm{DSC}}=\sum_{\mathbf{k} \sigma}\left(\epsilon_{\mathbf{k}}-\mu\right) c_{\mathbf{k} \sigma}^{\dagger} c_{\mathbf{k} \sigma}+\sum_{\mathbf{k}} \Delta_{\mathbf{k}} c_{\mathbf{k} \uparrow}^{\dagger} c_{-\mathbf{k} \downarrow}^{\dagger}+\text { h.c. }
$$

The spin-spin correlation function, at $\mu=0$ and momentum transfer $\mathbf{q}$, is

$$
\begin{aligned}
\mathcal{S}(\mathbf{q}, \omega, \mu=0) & =\frac{3 \pi}{2} \sum_{\mathbf{k} \in \mathrm{rbz}}\left(1-\frac{\epsilon_{\mathbf{k}} \epsilon_{\mathbf{k}+\mathbf{q}}+\Delta_{\mathbf{k}} \Delta_{\mathbf{k}+\mathbf{q}}}{E_{\mathbf{k}} E_{\mathbf{k}+\mathbf{q}}}\right) \\
& \times \delta\left(\omega-E_{\mathbf{k}}-E_{\mathbf{k}+\mathbf{q}}\right),
\end{aligned}
$$

where $E_{\mathbf{k}}=\sqrt{\Delta_{\mathbf{k}}^{2}+\epsilon_{\mathbf{k}}^{2}}$. For $\mathbf{q}=\mathbf{Q}$ the coherence factor equals two and Eq. 8 becomes identical to Eq. A. Hence, just like the DDW, at $\mu=0$ the DSC spin-spin correlator also shows a peak at twice the gap maximum, $2 \Delta_{0}$.

But for a DSC, at non-zero values of $\mu, \mathcal{S}(\mathbf{q}, \omega, \mu)$ is given by

$$
\begin{aligned}
\mathcal{S}(\mathbf{q}, \omega, \mu) & =\frac{3 \pi}{2} \sum_{\mathbf{k} \in \mathrm{rbz}}\left(1-\frac{\widetilde{\epsilon}_{\mathbf{k}} \widetilde{\epsilon}_{\mathbf{k}+\mathbf{q}}+\Delta_{\mathbf{k}} \Delta_{\mathbf{k}+\mathbf{q}}}{E_{\mathbf{k}} E_{\mathbf{k}+\mathbf{q}}}\right) \\
& \times \delta\left(\omega-E_{\mathbf{k}}-E_{\mathbf{k}+\mathbf{q}}\right)
\end{aligned}
$$

where $E_{\mathbf{k}}$ now incorporates $\mu$ in the usual way by $\epsilon_{\mathbf{k}} \rightarrow$ $\tilde{\epsilon}_{\mathbf{k}}=\epsilon_{\mathbf{k}}-\mu$. Equation 9 shows a peak at $\mathbf{q}=\mathbf{Q}$ that is shifted in energy from $2 \Delta_{0}$. The peak-energy shift is small for small $|\mu|$, but can be easily checked to scale with $2|\mu|$ as $|\mu|$ becomes large. Hence, one can clearly see that the peak-energy behaves differently with $\mu$ for DDW and DSC.
These Hartree-Fock calculations for the resonance peak in the normal state becom e interesting only if the chemical potential remains pinned close to $\mu=0$ in the underdoped regime. (This is not true for the calculation of the superfluid density reported in the following section, which is insensitive to such assumptions for $\mu$.) There is some evidence that this is the case in the photoemission measurements of Ino et al. [16] in $\mathrm{La}_{2-x} \mathrm{Sr}_{x} \mathrm{CuO}_{4}$. They find that while the chemical potential shift is large in the overdoped samples, it is largely suppressed in the underdoped regime. Numerical studies of the 2D Hubbard model 17,18 also suggest that the chemical potential does not shift much for small doping-fractions. In the Monte Carlo study performed in reference [17], the calculated shift of $\mu$ follows $\Delta \mu=\mu \propto-x^{2}$ for small values of doping, $x$. The data of reference [16] suggest that the chemical potential varies with $x$ in the overdoped regime as one would expect for a Fermi liquid, but not in the underdoped regime. Ideas involving charge ordered stripe states [19] are suggestive of the segregation of doped holes in the boundaries of antiferromagnetic domains, thus pinning the chemical potential. The presence of charge inhomogeneity, such as stripes [20, or impurity-disorder may pin the chemical potential in other multilayer high- $T_{c}$ cuprate systems as well.

\section{Coexisting DDW and DSC}

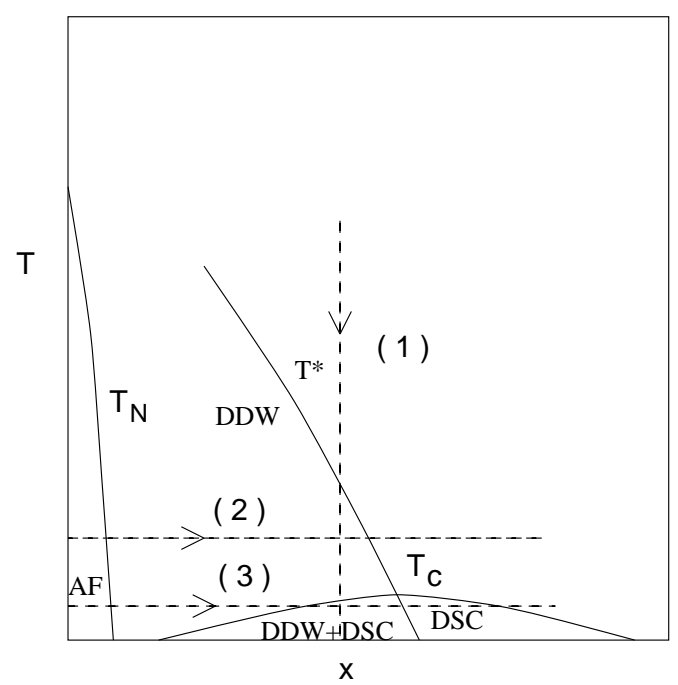

FIG. 1. The high- $T_{c}$ cuprate phase diagram proposed in Ref. 1. $T_{N}$ denotes the 3D- antiferromagnetic transition temperature. $T_{c}$ and $T^{*}$ are the DSC and pseudogap, or DDW, transition temperatures, respectively. In the text (Sec. V), the behavior of the spin-s pin correlator is discussed along the three paths shown here by the dashed lines.

From the order parameter competition picture pro- 
posed in reference [1] (See, Fig. 11), one notes that to the right of $x \simeq 0.05$ and below $T_{c}$, the two orders, singlet DDW and DSC, coexist and compete for the same regions on the Fermi surface. The mean-field Hamiltonian for the system in the folded Nambu basis [21] is

$$
H-\mu N=\sum_{\mathbf{k} \in \mathrm{rbz}} \Psi_{\mathbf{k}}^{\dagger} A_{\mathbf{k}} \Psi_{\mathbf{k}}
$$

where $\Psi_{\mathbf{k}}^{\dagger}=\left(c_{\mathbf{k} \uparrow}^{\dagger}, c_{\mathbf{k}+\mathbf{Q} \uparrow}^{\dagger}, c_{-\mathbf{k} \downarrow}, c_{-\mathbf{k}-\mathbf{Q} \downarrow}\right)$ and the matrix $A_{\mathrm{k}}$ is

$$
A_{\mathbf{k}}=\left(\begin{array}{cccc}
\left(\epsilon_{\mathbf{k}}-\mu\right) & i W_{\mathbf{k}} & \Delta_{\mathbf{k}} & 0 \\
-i W_{\mathbf{k}} & -\left(\epsilon_{\mathbf{k}}+\mu\right) & 0 & -\Delta_{\mathbf{k}} \\
\Delta_{\mathbf{k}} & 0 & -\left(\epsilon_{\mathbf{k}}-\mu\right) & i W_{\mathbf{k}} \\
0 & -\Delta_{\mathbf{k}} & -i W_{\mathbf{k}} & \left(\epsilon_{\mathbf{k}}+\mu\right)
\end{array}\right)
$$

The matrix $A_{\mathbf{k}}$ has four eigenvalues, $\pm E_{1}(\mathbf{k})$ and $\pm E_{2}(\mathbf{k})$, where $E_{1}(\mathbf{k})=\left[\left(\sqrt{\epsilon_{\mathbf{k}}^{2}+W_{\mathbf{k}}^{2}}-\mu\right)^{2}+\Delta_{\mathbf{k}}^{2}\right]^{1 / 2}$ and $E_{2}(\mathbf{k})=\left[\left(\sqrt{\epsilon_{\mathbf{k}}^{2}+W_{\mathbf{k}}^{2}}+\mu\right)^{2}+\Delta_{\mathbf{k}}^{2}\right]^{1 / 2}$.

The spin-spin correlation function is obtained by first evaluating the imaginary-time ordered correlator $\left(\omega_{m}\right.$ is the bosonic Matsubara frequency)

$$
\begin{aligned}
& \left\langle T_{\tau} \mathbf{S}\left(\mathbf{q}, i \omega_{m}\right) \cdot \mathbf{S}\left(-\mathbf{q},-i \omega_{m}\right)\right\rangle= \\
& \quad \frac{3}{4 \beta} \sum_{n, \mathbf{k} \in \mathrm{rbz}} \operatorname{Tr}\left[G\left(\mathbf{k}, i \omega_{n}\right) G\left(\mathbf{k}+\mathbf{q}, i \omega_{n}+i \omega_{m}\right)\right]
\end{aligned}
$$

where $\mathbf{S}$ denotes the spin operator and the $G$ 's are the $4 \times 4$ matrix Green functions computed from the Hamiltonian in Eq. 10. After analytically continuing the frequency to the real axis, we extract the imaginary part and use the fluctuation-dissipation theorem to get the spin-spin correlation function.

The zero-temperature spin-spin correlation function thus obtained, at non-zero $\mu$, for momentum transfer $\mathbf{q}=\mathbf{Q}$, is given by

$$
\begin{aligned}
\mathcal{S}(\mathbf{Q}, \omega, \mu)=\frac{3 \pi}{2} \sum_{\mathbf{k} \in \mathrm{rbz}} & \left(1+\frac{\epsilon_{\mathbf{k}}^{2}+\Delta_{\mathbf{k}}^{2}+W_{\mathbf{k}}^{2}-\mu^{2}}{E_{1}(\mathbf{k}) E_{2}(\mathbf{k})}\right) \\
\times & \delta\left(\omega-E_{1}(\mathbf{k})-E_{2}(\mathbf{k})\right)
\end{aligned}
$$

When $W_{\mathbf{k}}=0$, the coherence factor in Eq. 13 clearly matches with the coherence factor in Eq. 9, where we have to put $\mathbf{q}=\mathbf{Q}$, and the two expressions become identical. On the other hand, for $\Delta_{\mathbf{k}}=0$, one notes that $E_{1}(\mathbf{k}) \rightarrow\left|\sqrt{\epsilon_{\mathbf{k}}^{2}+W_{\mathbf{k}}^{2}}-\mu\right|$, and $E_{2}(\mathbf{k}) \rightarrow\left|\sqrt{\epsilon_{\mathbf{k}}^{2}+W_{\mathbf{k}}^{2}}+\mu\right|$. Keeping in mind that $\mu$ is negative in our hole-doped system, we note that for $\sqrt{\epsilon_{\mathbf{k}}^{2}+W_{\mathbf{k}}^{2}}+\mu>0$ the coherence factor in Eq. 13 is two, while the other choice for $\sqrt{\epsilon_{\mathbf{k}}^{2}+W_{\mathbf{k}}^{2}}+\mu<0$ reduces the coherence factor to zero. Thus the expression in Eq. 13 becomes identical to the expression in Eq. f for $\Delta_{\mathbf{k}}=0$.

One can easily check that $\mathcal{S}(\mathbf{Q}, \omega, \mu)$, as a function of $\omega$, has a peak in the spin-spin correlation function. The peak is located at $2 \sqrt{W_{0}^{2}+\Delta_{0}^{2}}$ for $\mu=0$. For small $\mu$ the peak-energy is shifted to higher values, initially by a small amount, but finally scaling as $2|\mu|$ for large values of $|\mu|$. We should mention here that one can in fact control the behavior of the peak-energy with $\mu$ by adjusting the relative strengths of the order parameters. When the singlet DDW order overshadows the DSC order, the peak-energy will tend to be pinned to the total order parameter magnitude $2 \sqrt{W_{0}^{2}+\Delta_{0}^{2}}$, a behavior characteristic of the singlet DDW.

\section{RPA Corrections}

If we add a reduced Coulomb repulsion $\bar{U}$, which is assumed to be renormalized due to particle-particle correlations, we can drive the system toward antiferromagnetism. To model this effect, we have to go beyond the Hartree-Fock, and we use a crude RPA form [1] for the susceptibility $\chi(\mathbf{q}, \omega)$ given by,

$$
\chi(\mathbf{q}, \omega)=\frac{\chi_{0}(\mathbf{q}, \omega)}{1-\bar{U} \chi_{0}(\mathbf{q}, \omega)}
$$

to describe the spin dynamics in the system. Here $\chi_{0}(\mathbf{q}, \omega)$ is the Hartree-Fock susceptibility. Extracting $\mathcal{S}(\mathbf{q}, \omega, \mu)$ from $\chi(\mathbf{q}, \omega, \mu)$, in Fig. 22 we plot the results as a function of frequency for five different values of $\bar{U}$.

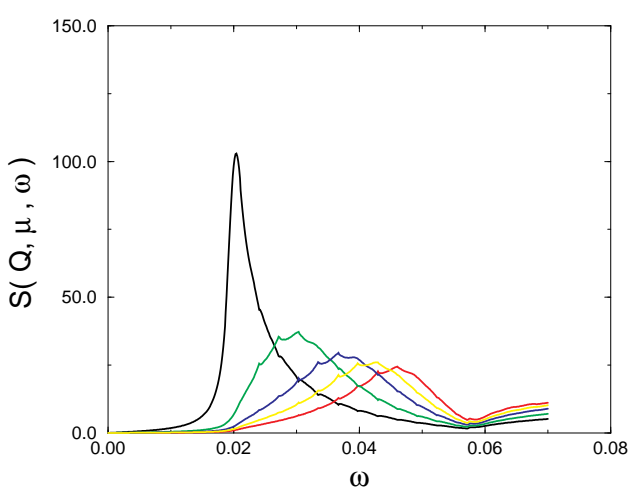

FIG. 2. $\mathcal{S}(\mathbf{Q}, \omega, \mu)$ as a function of $\omega$ after $\bar{U}$ is introduced into the system with coexisting singlet DDW and DSC orders with $t=0.5 \mathrm{eV}, \Delta_{0}=.02 \mathrm{eV}, W_{0}=.02 \mathrm{eV}$ and $\mu$ set to $-.01 \mathrm{eV}$. As $\bar{U}$ is increased, the structures are peaked at lower and lower energies; from right to left $\bar{U}=0.2,0.217,0.233,0.256,0.284$. Finally they evolve, as $\bar{U}=\bar{U}_{c}=0.32 \mathrm{eV}$ is approached, into a divergence at zero energy.

The first thing one notices is that after the introduction of $\bar{U}$, the correlation function $\mathcal{S}$, which was logarithmically singular when derived from $\chi_{0}(\mathbf{q}, \omega)$ alone, now becomes broad. Also, as $\bar{U}$ is progressively increased, 
the spectral-weight appears at smaller and smaller energies. For $\bar{U}$ large enough, the correlator starts peaking up again and finally evolves into a divergence at zero energy as $\bar{U}_{c}$ is approached. All of this behavior is qualitatively consistent with what happens to the spinfluctuation spectrum as the doping is reduced in the underdoped regime of the cuprates. As the hole density is reduced, the repulsive Coulomb energy becomes more and more important and gradually approaches the critical value $\bar{U}_{c}$ for which the peak in the spin-spin correlation function evolves into a divergence at zero energy, signalling antiferromagnetic order in the system.

This analysis applies both below and above $T_{c}$ in the high- $T_{c}$ cuprate materials. Above the superconducting transition temperature $T_{c}, \Delta_{\mathbf{k}}$ will go to zero, but $W_{\mathbf{k}}$ will survive up to the pseudogap temperature $T^{*}$. Hence, in the spin-spin correlator the DDW will continue to maintain its signature intact in the form of a peak at twice the maximum of its gap.

\section{SUPERFLUID DENSITY}

The anomalous behavior of the superfluid density in the underdoped regime of the high- $T_{c}$ cuprates has been discussed extensively. Here, we address it in the context of the competition of the order parameters that we have been discussing 22. We will make a phenomenological assumption about the doping dependence of the DDW and single particle gaps, respectively, $W_{\mathbf{k}}$ and $\sqrt{\Delta_{\mathbf{k}}^{2}+W_{\mathbf{k}}^{2}}$. Following [23], we hypothesize that the DDW order parameter vanishes above a critical doping $x_{c}$ (about 0.2) and follows the formula $W_{0} / k_{B} \simeq$ $\alpha J^{*}\left(1-x / x_{c}\right)$ where $J^{*}=980 K, x$ is the doping-fraction, and $\alpha \simeq 0.6$ is a constant arbitrarily chosen to set a scale. In other words,

$$
W_{0} \simeq .049\left(1-x / x_{c}\right)
$$

in $\mathrm{eV}$; in Fig. 3, we test another model in which $W_{0} \propto$ $\left(1-x / x_{c}\right)^{2}$.

We also posit that the single-particle gap remains constant at its value at $x=.05$, where superconductivity starts developing; then

$$
W_{0}^{2}+\Delta_{0}^{2} \equiv \Phi_{0}^{2}=(.037)^{2}
$$

We calculate the superfluid stiffness for such a model using the formula 24]

$$
\frac{n_{s}}{m^{*}}=\left\langle-k_{x}\right\rangle-\Lambda_{x x}\left(q_{x}=0, q_{y} \rightarrow 0, i \omega_{m}=0\right)
$$

which gives the response to a transverse vector potential $A_{x}(t)$. Here, $n_{s}$ is the superfluid density and $m^{*}$ is the effective mass, $\left\langle k_{x}\right\rangle$ is the kinetic energy per site per lattice dimension, and $\Lambda_{x x}\left(\mathbf{q}, i \omega_{m}\right)$ is the paramagnetic current-current correlation function.
The paramagnetic current response is obtained from the relation

$$
\begin{aligned}
\Lambda_{x x}\left(\mathbf{q}, i \omega_{m}\right)= & \left\langle T_{\tau} j_{x}\left(\mathbf{q}, i \omega_{m}\right) j_{x}\left(-\mathbf{q},-i \omega_{m}\right)\right\rangle \\
= & \frac{4 t^{2}}{\beta} \sum_{n, \mathbf{k} \in \mathrm{rbz}} \sin k_{x} \sin (\mathbf{k}+\mathbf{q})_{x} \\
& \operatorname{Tr}\left[G\left(\mathbf{k}, i \omega_{n}\right) M G\left(\mathbf{k}+\mathbf{q}, i \omega_{n}+i \omega_{m}\right) M\right] \\
& -\frac{4 W_{0}^{2}}{\beta} \sum_{n, \mathbf{k} \in \mathrm{rbz}} \sin k_{x} \sin (\mathbf{k}+\mathbf{q})_{x} \\
& \operatorname{Tr}\left[G\left(\mathbf{k}, i \omega_{n}\right) N G\left(\mathbf{k}+\mathbf{q}, i \omega_{n}+i \omega_{m}\right) N\right]
\end{aligned}
$$

In Eq. 18, the $G$ 's are the same matrix Green functions used in the calculation of the spin-spin correlation function and $M$ and $N$ are the matrices

$$
M=\left(\begin{array}{cccc}
1 & 0 & 0 & 0 \\
0 & -1 & 0 & 0 \\
0 & 0 & 1 & 0 \\
0 & 0 & 0 & -1
\end{array}\right)
$$

$$
N=\left(\begin{array}{cccc}
0 & 1 & 0 & 0 \\
-1 & 0 & 0 & 0 \\
0 & 0 & 0 & -1 \\
0 & 0 & 1 & 0
\end{array}\right)
$$

The superfluid stiffness (and therefore the inverse square penetration depth) at $T=0$, evaluated in the entire doping range, is given by

$$
\begin{aligned}
\frac{n_{s}}{m^{*}} & =-32 t^{2} \sum_{\mathbf{k} \in \mathrm{rbz}} \sin ^{2} k_{x} W_{\mathbf{k}}^{2}\left(1+\frac{\epsilon_{\mathbf{k}}^{2}+\Delta_{\mathbf{k}}^{2}+W_{\mathbf{k}}^{2}-\mu^{2}}{E_{1}(\mathbf{k}) E_{2}(\mathbf{k})}\right) \\
& \times \frac{1}{\left[E_{1}(\mathbf{k})+E_{2}(\mathbf{k})\right]^{3}}\left(1+\mathcal{O}\left(W_{0}^{2} / t^{2}\right)\right) \\
& +8 t^{2} \lim _{\beta \rightarrow \infty} \sum_{\mathbf{k} \in \mathrm{rbz}} \sin ^{2} k_{x} \frac{\epsilon_{\mathbf{k}}^{2}}{W_{\mathbf{k}}^{2}+\epsilon_{\mathbf{k}}^{2}} \\
& \times\left(\frac{d f\left(E_{1}(\mathbf{k})\right)}{d E_{1}(\mathbf{k})}+\frac{d f\left(E_{2}(\mathbf{k})\right)}{d E_{2}(\mathbf{k})}\right)\left(1+\mathcal{O}\left(W_{0}^{2} / t^{2}\right)\right) \\
& -4 t \sum_{\mathbf{k} \in \mathrm{rbz}} \cos k_{x}\left(1+\frac{\epsilon_{\mathbf{k}}^{2}+\Delta_{\mathbf{k}}^{2}+W_{\mathbf{k}}^{2}-\mu^{2}}{E_{1}(\mathbf{k}) E_{2}(\mathbf{k})}\right) \\
& \times \frac{\epsilon_{\mathbf{k}}}{E_{1}(\mathbf{k})+E_{2}(\mathbf{k})}\left(1+\mathcal{O}\left(W_{0}^{2} / t^{2}\right)\right)
\end{aligned}
$$

where $E_{1}(\mathbf{k})$ and $E_{2}(\mathbf{k})$ are the two energy-values given before. The first two terms in Eq. 19 come from the paramagnetic current response, and the last term is the kinetic energy (or the diamagnetic term). At finite, but low temperatures, one can show that

$$
\begin{aligned}
\frac{n_{s}}{m^{*}}(T) & -\frac{n_{s}}{m^{*}}(0) \approx 8 t^{2} \sum_{\mathbf{k} \in \mathrm{rbz}} \sin ^{2} k_{x} \frac{\epsilon_{\mathbf{k}}^{2}}{W_{\mathbf{k}}^{2}+\epsilon_{\mathbf{k}}^{2}} \\
& \times\left(\frac{d f\left(E_{1}(\mathbf{k})\right)}{d E_{1}(\mathbf{k})}+\frac{d f\left(E_{2}(\mathbf{k})\right)}{d E_{2}(\mathbf{k})}\right)\left(1+\mathcal{O}\left(W_{0}^{2} / t^{2}\right)\right) .
\end{aligned}
$$


The $\mathcal{O}\left(W_{0}^{2} / t^{2}\right)$ terms derive from the modification of both the kinetic energy and current operators in the lowenergy effective (Hartree-Fock) Hamiltonian associated with DDW order. The modified current operator gives rise to the matrix $N$ in (18). Since these terms are small, $\mathcal{O}\left(W_{0}^{2} / t^{2}\right)$, we neglect them in the following discussion.

Equation 19 has several interesting properties. First, for $\Delta_{\mathbf{k}}=\Delta_{0} \neq 0$ and $W_{\mathbf{k}}=W_{0}$, that is, for competing CDW and $s$-wave superconductivity, the terms involving the Fermi functions do not contribute at zerotemperature. At $\mu=0, E_{1}(\mathbf{k})=E_{2}(\mathbf{k})$, and the same coherence factor in the other two terms becomes two. Then, by a partial integration one can show that (denoting the first term by $K^{\text {para }}$ and the third term by $\left.K^{\text {dia }}\right), K^{\text {para }}=-\frac{W_{0}^{2}}{\Phi_{0}^{2}} K^{\text {dia }}$, and the full kernel is given by $K^{\text {total }}=K^{\text {para }}+K^{\text {dia }}=K^{\text {dia }}\left(1-\frac{W_{0}^{2}}{\Phi_{0}^{2}}\right)$. When the two orders are of $s$-wave type [15], $K^{\text {total }}$ yields a superfluid density which is maximum when $W_{0}=0$ (i.e, $\Delta_{0}=\Phi_{0}$ ) and zero when $W_{0}=\Phi_{0}\left(i . e, \Delta_{0}=0\right)$ [25].

Returning to $d$-wave order parameters, at $\Delta_{0}=0$, Eq. 19 reduces to the superfluid density of the DDW state, which is, of course, zero. The second term, which contains the derivatives of the Fermi functions is crucial for the cancellation in this case. For $\Delta_{0}$ and $\mu$ finite, the second term does not contribute at zero temperature (except for a contribution from a single point in k-space; but the finite and almost constant contribution from this is ignored here for simplicity). The system now acquires a finite superfluid density.

The superfluid density derived from Eq. 19 can be cast as a function of a single variable, $x$, by expressing the three parameters $W_{0}, \Delta_{0}$ and $\mu$ in terms of the doping fraction. For the dependence of $\mu$ on $x$, we use $\mu \simeq-x^{2}$ (in units in which $2 t=1$ ) as in reference [17], but other reasonable dependences should give similar results ( $c f$. the discussion in the previous section). Hence, the results of this section are valid and relevant for the cuprates even if $\mu$ is not pinned close to zero in the underdoped regime. For $W_{0}$ and $\Delta_{0}$ we choose the relations given in Eq. 15 and Eq. 16 only because they have received some recent experimental support [23]. But it's worthemphasizing that the qualitative features that we extract from Eq. 19 for the behavior of the superfluid density with $x$ are fairly independent of the precise functional forms of $W_{0}$ and $\Delta_{0}$. The only input that is needed is the existence of DDW order, with diminishing strength with $x$, and complementary development of the DSC order. The DDW order eats away part of the superfluid density from an otherwise pure DSC system even within the superconducting dome in the $T-x$ phase diagram. With increasing $x$, the DDW order weakens, hence the superfluid density increases in a model-independent manner.

In Fig. 3 we plot $n_{s} / m^{*}$ against the doping-fraction $x$ using Eq. 15 and Eq. 16 for $W_{0}$ and $\Delta_{0}$. To illustrate the qualitative robustness of our result, we also show, by the dashed curve, the same plot for $W_{0}$ dispersing quadratically with $x$ between the same two end points as in Eq. 15. As can be seen from the figure the zerotemperature superfluid density, in a model-independent manner, shows a rapid drop in the underdoped regime, similar to that observed in experiments.

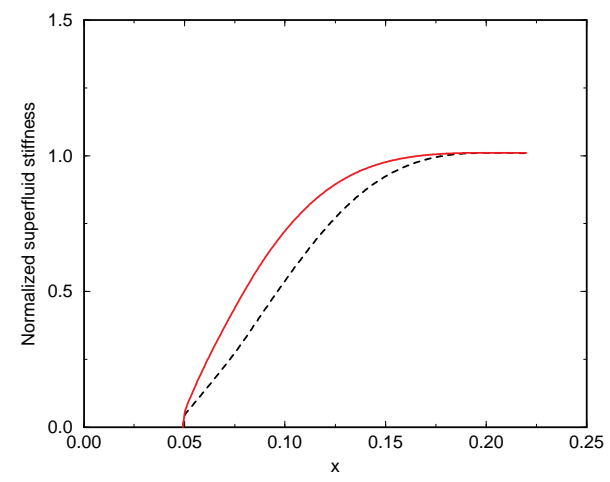

FIG. 3. The $T=0$ superfluid stiffness $n_{s} / m^{*}$ as a function of doping fraction $x$. The vertical axis is normalized by the superfluid stiffness at $x=0.2$. Here, we have set $t=0.5 \mathrm{eV}$ and the chemical potential is taken to be $\mu=-x^{2}$. The amplitudes of the order parameters are as given in Eqs. 15 and 16. The dashed curve is $n_{s} / m^{*}$ for $W_{0}$ dispersing quadratically with $x$ as explained in the text.

The leading temperature dependence of the superfluid stiffness can be evaluated from Eq. 20. At temperatures much smaller than the relevant energy scales $W_{0}$ and $\Delta_{0}$, only the nodal regions close to the points $(\pi / 2, \pi / 2)$ and symmetry-related points will contribute in the suppression of the superfluid weight. By expanding around those points, one can see that the leading temperature dependence is indeed linear for the optimally and moderately doped samples in a fairly wide range of temperatures. For these doping concentrations, where $\Delta_{0}$ is larger than or comparable to $W_{0}, W_{0}$ plays a subleading role to $\Delta_{0}$ in determining the temperature dependence of the suppression. This is due to the peculiar band structure of the problem.

On the other hand, for the heavily underdoped samples the situation is quite different. Critical fluctuations are clearly important to determine any low-temperature property of the system, for it is close to a quantum critical point. Even if we ignore these fluctuations, and rely strictly on our mean-field results, the conclusions are very different from moderate or optimally doped samples. Though in the asymptotically low temperature regime, the depletion of the superfluid density is linear in temperature, there is an intermediate temperature range over which the suppression of the superfluid density actually 
behaves as $\sqrt{T}$. As the DDW gap is much larger than the superconducting gap in these heavily underdoped samples, $W_{0}$ crosses over to produce the leading contributions in the expansion of Eq. 20 around the nodes, and is eventually responsible for the $\sqrt{T}$ behavior of the suppression. All of this is summarized in Fig. 4, where we have plotted the temperature-dependent part of the superfluid stiffness with temperature scaled by the superconducting gap, for six values of the doping fraction $x$. The plot shows $\sqrt{T}$ behavior for the heavily underdoped systems for the experimentally relevant temperatures [12]. For asymptotically low temperatures for these doping values, and for a fairly wide range of temperatures for moderate or optimal doping, the data show exact collapse on a single straight line signifying a unique low-temperaure slope.

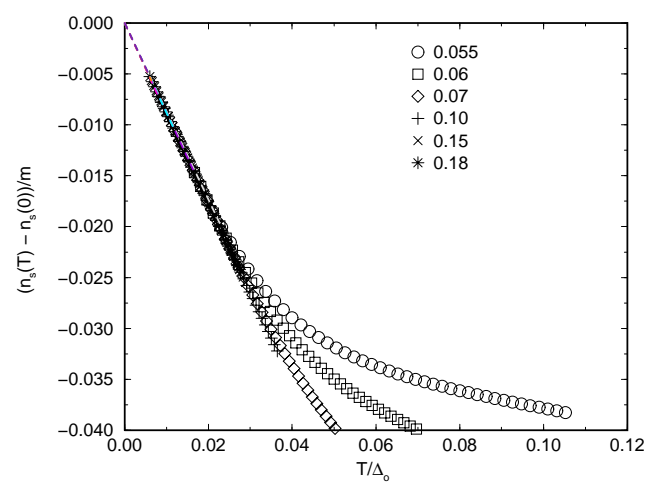

FIG. 4. The temperature-dependent part of the superfluid stiffness plotted against scaled temperature for six values of the doping. The doping concentrations are indicated in the legends. The values of $W_{0}$ and $\Delta_{0}$ for a given doping are derived from Eqs. 15 and 16. $\mu$ is derived from $\mu=-x^{2}$ and $t=0.5 \mathrm{eV}$. The dotted li ne is an extrapolation of the results to zero temperature.

\section{DISCUSSION}

The cuprate phase diagram proposed in reference [1] is reproduced here in Fig. 11 for easy reference; we only show the relevant portion of the phase diagram, ignoring the complex set of competing charge ordered states in the underdoped regime as well as the spin glass phase.

From our calculation, the behavior of the $T=0 \mathrm{su}-$ perfluid density with $x$ is as expected from experiments in the underdoped regime of the high- $T_{c}$ cuprates. The striking rapid suppression of the superfluid density (and sharp increase of the London penetration depth) below $x \simeq 0.2$ is naturally explained by the emergence of the singlet DDW at that doping-fraction. The behavior of the superfluid density near $T_{c}$ or near $x \approx 0.05$ (the lowest doping at which superconductivity occurs) will be strongly affected by thermal or quantum phase fluctuations, so our Hartree-Fock results will be suspect in those regimes. However, away from these critical points, we might expect our calculation to be on solid footing, and it is encouraging to see that it agrees with experimental measurements of $n_{s}$. The temperature dependence also captures the striking linearity shown in Fig. 4, and the doping independence of the slope, along with a $\sqrt{T}$ dependence in an intermediate temperature regime in the heavily underdoped regime akin to the experiments [12].

The neutron scattering peaks observed in the pseudogap and superconducting regimes are broadly consistent with our calculations. There are peaks at $(\pi, \pi)$ at energies which are controlled by the single-particle gap and the doping. There are no observable peaks at $(\pi, 0)$ and symmetry-related points because, from our analysis, they would be at high energies controlled by the band dispersion where they are likely to be strongly damped. This resolves an earlier puzzle of the analysis of the staggered flux phase 14. Though we have not presented here results for finite tempeartures, from the phase diagram and our zero temperature theory, the qualitative aspects at finite temperatures can still be explained. However, the details of the peak position in energy and its doping dependence is beyond a simple Hartree-Fock calculation.

If one approaches along the path labeled (1) in Fig. 1, at first one will find no structure in the spin-spin correlator, typical of a Fermi liquid. Below $T^{*}$, the correlator peaks at an energy $2 W_{0}$, twice the maximum of the DDW gap at the wavevector $\mathbf{Q}$. This is consistent with experiments. Below $T_{c}$, the intensity in the spin-spin correlator is amplified as the DSC order develops, as discussed in Ref. [3]. Due to the coexistence of $D D W$ and $D S C$, the peak will be at a higher energy $-2 \sqrt{W_{0}^{2}+\Delta_{0}^{2}}$, shifted by an amount that depends on the doping. To see this, recall that, for coexisting DDW and DSC, the peak-energy shifts to higher values with $|\mu|$, which increases with doping.

Consider now the path (2) in Fig. 1. In the DDW state, and for small chemical potential, the neutron scattering intensity should exhibit a peak at wavevector $\mathbf{Q}$ and energy $2 W_{0}$; recall that, for DDW order, the peakenergy shows no movement with $|\mu|$, but is destroyed by a large $|\mu|>W_{0}$. Since the DDW gap varies with $x$ as in Eq. 15, the peak would shift to higher energies as $x$ is decreased, if $\chi_{0}$ alone were responsible for the spinfluctuation spectrum. However, the reduced Coulomb interaction $\bar{U}$ is important since it shifts the peaks to lower energies, broadening them simultaneously. For smaller $x$, due to the lower density of the holes, the effective $\bar{U}$ increases, and, as a result, as shown in Fig. 2, the peak moves to lower energies. Consequently, the peak-energies in this part of the phase diagram would be influenced by these two competing effects. 
Consider the path (3) in Fig. 1. In the coexisting region, DDW plus DSC, the peak energy is $2 \sqrt{W_{0}^{2}+\Delta_{0}^{2}}$, shifted by $|\mu|$, but now, in contrast to $T>T_{c}$, the magnitude of the total order parameter remains constant as per Eq. 16. Of course, $\bar{U}$ must be important as well, modifying this conclusion.

\section{ACKNOWLEDGMENTS}

We would like to thank Qiang-Hua Wang for pointing out a minor error in an earlier version of this paper. S. C. and S. T. are supported by NSF-DMR-9971138, and C. N. by NSF-DMR-9983544 and the A.P. Sloan Foundation. The work of H. -Y. K. was conducted under the auspices of the DOE, supported by funds provided by the University of California for the conduct of discretionary research by Los Alamos National Laboratory.

[1] S. Chakravarty, R. B. Laughlin, D. K. Morr, and C. Nayak, Phys. Rev. B 63, 94503 (2001).

[2] C. Nayak, Phys. Rev. B 62, 4880 (2000); ibid, R6135 (2000); C. Nayak and F. Wilczek, cond-mat/9510132

[3] S. Chakravarty and H.-Y. Kee, Phys. Rev. B 61, 14821(2000).

[4] H. A. Mook, P. Dai, F. Dogan, cond-mat/0102047; H. A. Mook, Pengcheng Dai, S. M. Hayden, A. Hiess, and F. Dogan, "Polarized neutron beam observation of antiferromagnetic order in $\mathrm{YBa}_{2} \mathrm{Cu}_{3} \mathrm{O}_{6.6}$ ", preprint. The data of Y. Sidis et al., Phys. Rev. Lett. 86, 4100 (2001), on lower doping material $\mathrm{YBCO}_{6.5}$ are entirely different and appear to reflect remnant spin magnetism instead. There is no contradiction in this, because DDW order can disappear as the doping is reduced; see S. Chakravarty, H. -Y. Kee and C. Nayak, cond-mat/0101204.

[5] J. E. Sonier, J. H. Brewer, R. F. Kiefl, R. I. Miller, G. D. Morris, C. E. Stronach, J. S. Garner, S. R. Dunsiger, D. A. Bonn, W. N. Hardy, R. Liang, R. H. Heffner, Science, to be published.

[6] M. V. Eremin et al., cond-mat/0105508.

[7] For recent reviews, see H. F. Fong et al., Phys. Rev. B 61, 14773 (2000); P. Dai et al., Phys. Rev. B 63, 054525 (2001).

[8] For a cross section of theoretical papers on the neutron resonance peak, see K. Maki and H. Won, Phys. Rev. Lett. 72, 1758 (1994); M. J. Lercher and J. M. Wheatley, Phys. Rev. Lett. B 49, 736 (1994); M. Lavagna ang G. Stenmann, Phys. Rev. B 49, 4235 (1994); G. Stenmann and C. Pépin, and M. Lavagna, Phys. Rev B 50, 4075 (1994); F. Onufrieva and J. Rossat-Mignod, Phys. Rev. B 52, 7572 (1995); G. Blumberg, B. P. Stojkovic, and M. V. Klein, Phys. Rev. B 52, 15741 (1992); A. A. Abrikosov, Phys. Rev. B 57, 8656 (1998); L. Yin, S. Chakravarty, and P. W. Anderson, Phys. Rev. Lett. 78, 3559 (1997);
N. Bulut and D. J. Scalapino, Phys. Rev. B 53, 5149 (1996); D. Z. Liu, Y. Zha, and K. Levin, Phys. Rev. Lett. 75, 4130 (1995); I. I. Mazin and V. M. Yakovenko, Phys. Rev. Lett. 75, 4134 (1995); J. Brinckmann and P. A. Lee, Phys. Rev. Lett. 82, 2915 (1999); Y. J. Kao, Q. Si, and K. Levin, Phys. B 61, 11898 (2000); D. K. Morr and D. Pines, Phys. Rev. Lett. 81, 1086 (1998); A. J. Millis and H. Monien, Phys. Rev. B 54, 16172 (1996); T. Tanamoto, H. Kohno, and H. Fukuyama, J. Phys. Soc. Jpn. 61, 1886 (1992); B. Normand, H. Kohno, and H. Fukuyama, J. Phys. Soc. Jpn. 64, 3903 (1995); A. Abanov and A. V. Chubukov, Phys. Rev. Lett. 83, 1652 (1999); S. Sachdev, C. Buragohain, and M. Vojta, Science 286, 2479 (1999); E. Demler and S. C. Zhang, Phys. Rev. Lett. 75, 4126 (1995); E. Demler, H. Kohno, and S. C. Zhang, Phys. Rev. B 58, 5719 (1998).

[9] Pengcheng Dai, H. A. Mook, G. Aeppli, S. M. Hayden, and F. Dogan, Nature 406, 965 (2000).

[10] J.L. Tallon and J.W. Loram, Physica C 349, 53 (2001), cond-mat/0005463; J.W.Loram et al., J. Phys. Chem. Solids 59,2091 (1998); J.L.Tallon et al., Phys. Stat. Sol. B 215, 531 (1999).

[11] Y. J. Uemura et al., Phys. Rev. Lett, 62, 2317 (1989).

[12] C. Panagopoulos, J. L. Tallon, and T. Xiang, Phys. Rev. B 5 9, R6635 (1999); see also D. Brown, Ph. D. thesis, Cambridge University, unpublished (2000).

[13] E. Cappelluti and R. Zeyher, Phys. Rev. B 59, 6475 (1999); E. Cappelluti and R. Zeyher, Physica C 312, 313 (1999); E. Cappelluti and R. Zeyher, Europhys. Lett. 49, 487 (2000); R. Zeyher, Physica C 341,121 (2000).

[14] I. Affleck and J. B. Marston , Phys. Rev. B, 37, 3774 (1988); J. B. Marston and I. Affleck, Phys. Rev. B, 39, 11538 (1989).

[15] E. Fradkin, Field theories of condensed matter systems (Addison-Wesley, Redwood City,1991).

[16] A. Ino et al., Phys. Rev. Lett, 79, 2101 (1997)

[17] N. Furukawa and M. Imada, J. Phys. Soc. Jpn. 62, 2557 (1993)

[18] E. Dagotto et al., Phys. Rev. Lett, 67, 1918 (1991).

[19] See, for example, S. A. Kivelson and V. J. Emery, in "Strongly Correlated Electronic Materials: The Los Alamos Syposium 1993," edited by K. S. Bedell et al (Addison Wesley, Reading, 1994) p. 619.

[20] V. J. Emery, S. A. Kivelson, and O. Zachar, Phys. Rev. B 56, 6120, (1997).

[21] J.R.Schrieffer, The Theory of Superconductivity (W. A. Benjamin, Inc., Reading,1964). We fold the usual Nambu-basis, which is defined in the full Brillouin zone, onto the reduced Brillouin zone to incorporate DDW simultaneously with DSC; see also F. C. Zhang, Phys. Rev. Lett. 64, 974 (1990).

[22] See also a related preprint, Q. -H. Wang et al., condmat/0011398.

[23] J. W. Loram et al., Physica C, Feb 20-25, 2000.

[24] D. J. Scalapino et al., Phys. Rev. B, 47, 7995, (1993).

[25] P. Miller et al., Physica C, 210, 343 (1993); G. Bilbro and W. L. McMillan, Phys. Rev. B, 14, 1887 (1976). 